\title{
Xenon Doping of Liquid Argon
}

\author{
Alaina M. Swanson, University of Minnesota-Morris, SULI Program \\ Carlos O. Escobar, Fermi National Accelerator Laboratory
}

UNIVERSITY OF MINNESOTA

MORRIS

Doping liquid argon with xenon will shift the peak scintillation wavelength towards longer wavelengths. Tests were performed on photon detectors, light absorbing materials, and other equipment in preparation for an experiment on the scintillation of liquid argon doped with xenon.

\section{Motivation}

Liquid argon is an excellent medium for scintillation because it is inexpensive, has high scintillation efficiency, and can be purified easily. When an ionizing particle travels through liquid argon, it excites the argon and gives off scintillation light. This light has a wavelength of $128 \mathrm{~nm}$, which is too small for most photon detectors to detect. Doping argon with xenon will shift the wavelength of the light, because xenon emits a photon at $175 \mathrm{~nm}$.

Xenon doped argon is more efficient than other wavelength shifters because it is volume distributed. This method of wavelength shifting simplifies data collection and analysis because it improves light collection in large detectors, such as the Deep Underground Neutrino Experiment (DUNE).

$$
l^{-1}=\frac{16 \pi^{3}}{6 \lambda^{4}}\left[k T \rho \kappa_{T}\left(\frac{\left(n^{2}-1\right)\left(n^{2}+2\right)}{3}\right)^{2}\right]
$$

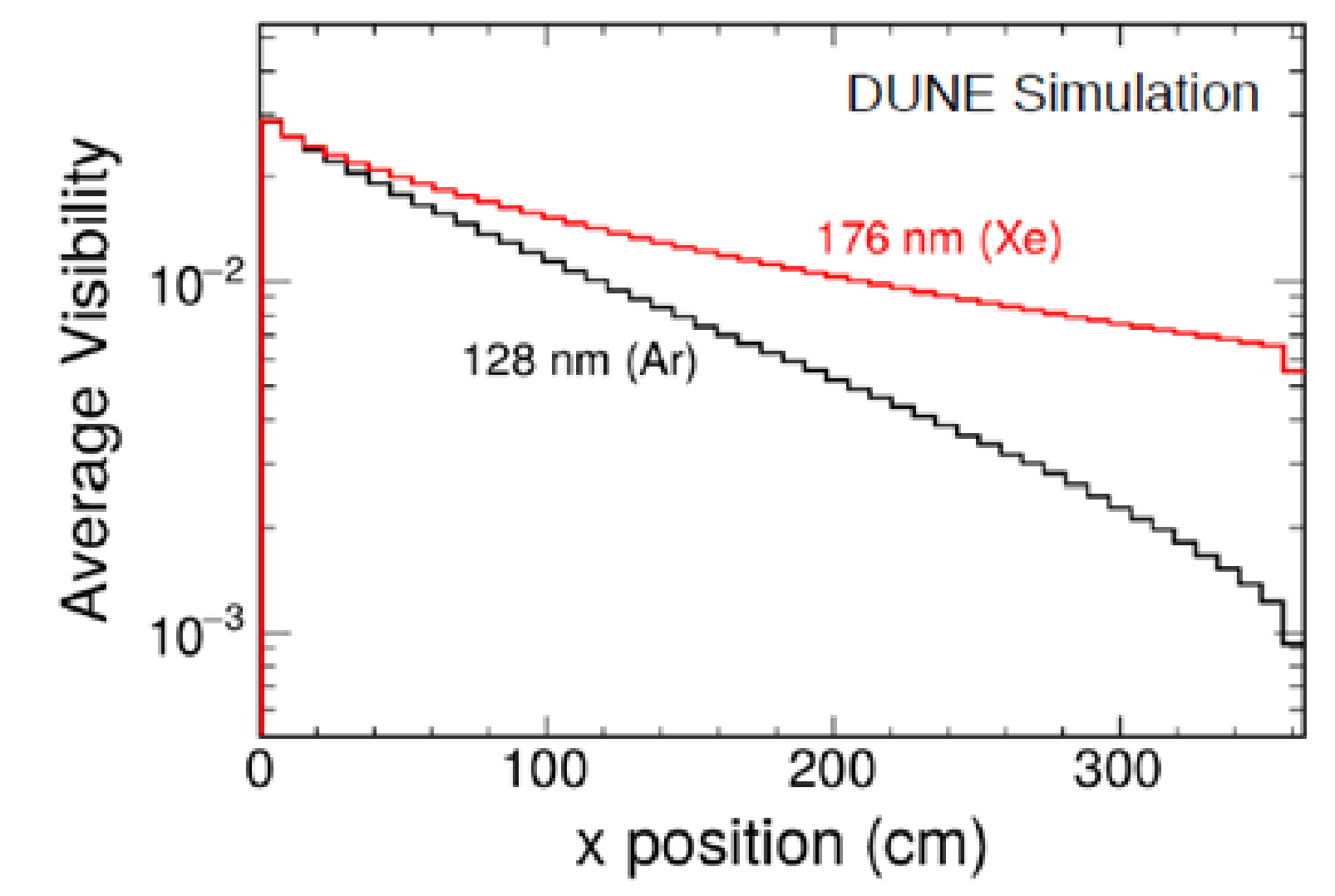

\section{Kinetics}

Rate of Argon Light Output $=\lambda_{A r, 1} N_{0} q e^{-\lambda_{1 m} t}+\lambda_{A r, 3} N_{0}(1-q) e^{-\lambda_{3 m} t}$

Rate of Xenon Light Output $=\frac{\lambda_{m} \lambda_{d} N_{0}\left(p \lambda_{d}\left(\lambda_{X e, 3}-\lambda_{X e, 1}\right)+\lambda_{X e, 3}\left(\lambda_{X e, 1}-\lambda_{d}\right)\right)\left((1-q) \lambda_{1 m}+q \lambda_{3 m}-\lambda_{d}\right)}{\left(\lambda_{d}-\lambda_{3 m}\right)\left(\lambda_{d}-\lambda_{1 m}\right)\left(\lambda_{X e, 1}-\lambda_{d}\right)\left(\lambda_{X e, 3}-\lambda_{d}\right)} e^{-\lambda_{d} t}$

$+\frac{\lambda_{d} \lambda_{m}\left(p \lambda_{1 m}\left(\lambda_{X e, 3}-\lambda_{X e, 1}\right)+\lambda_{X e, 3}\left(\lambda_{X e, 1}-\lambda_{1 m}\right)\right) q N_{0}}{\left(\lambda_{d}-\lambda_{1 m}\right)\left(\lambda_{X e, 1}-\lambda_{1 m}\right)\left(\lambda_{X e, 3}-\lambda_{1 m}\right)} e^{-\lambda_{1 m} t}+\frac{\lambda_{d} \lambda_{m}\left(p \lambda_{3 m}\left(\lambda_{X e, 3}-\lambda_{X e, 1}\right)+\lambda_{X e, 3}\left(\lambda_{X e, 1}-\lambda_{3 m}\right)\right)(1-q) N_{0}}{\left(\lambda_{d}-\lambda_{3 m}\right)\left(\lambda_{X e, 1}-\lambda_{3 m}\right)\left(\lambda_{X e, 3}-\lambda_{3 m}\right)} e^{-\lambda_{3 m} t}$

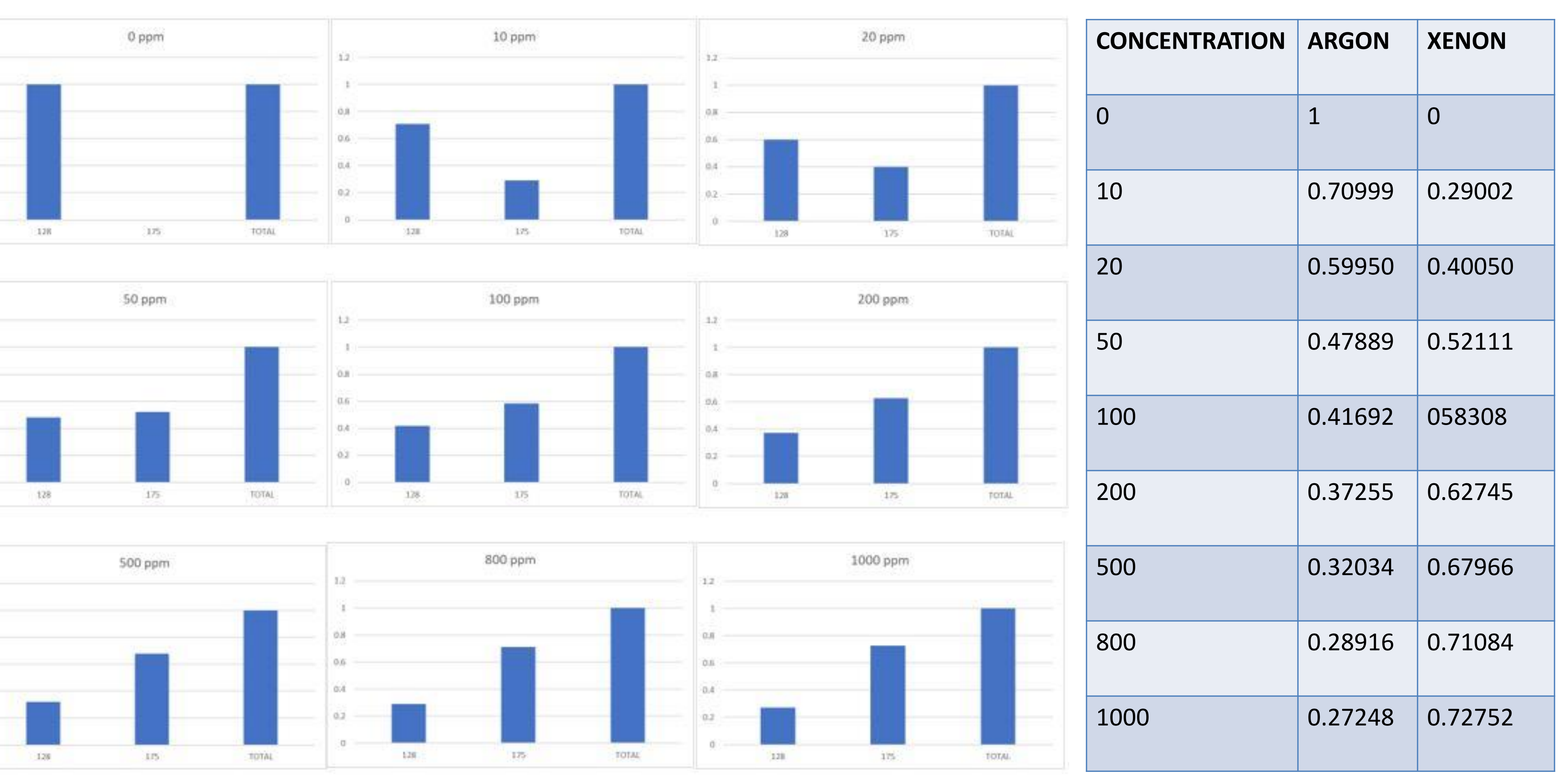

These results were obtained by integrating equations for light output for argon and xenon over a time interval from zero to infinity.

\section{Conclusion}

The results obtained from testing SiPMs \#33 and \#37 helped develop a better understanding of their characteristics. A significant amount of light was observed in the liquid argon using an alpha source. The breakdown voltages were found, which establishes a lower threshold for the voltage range.

The purity testing of the MetalVelvet sample proved that it is safe to use in the main experiment. The sample only fell to a lifetime of $1 \mathrm{~ms}$, which is not detrimental.

The graphs of the amount of wavelength shift per concentration establish a clear rubric for how much xenon dopant needs to be mixed with the argon. The graphs provide a visual key for determining the appropriate concentration.

\section{References}

[1] Akimov, D., Belov, V., Konovalov, A. \& Kumpan, A. Fast component re-emission in Xe-doped liquid argon. Journal of Instrumentation (2019).

[2] Buzulutskov, A. Photon emission and atomic collision processes in two-phase argon doped with xenon and nitrogen. Europhysics Letters 117, (2017)

4] Hitachi, A.: Photon-mediated and collisional processes in liquid rare gases. Nuclear Instruments and Methods in Physics Research Section A: Accelerators, Spectrometers, Detectors and Associated Equipment. 327, 11-14 (1993).

(G) Koll, G.F. Radiation Detection and Measurement. John Wiley and Sons Inc. (2010).

7] MetalVelvet coating: Optical black coating \& Ruan, J. The suppression of the slow component in x

8] Neumeier, A., Dandl, T., Heindl, T., Himpsl, A. \& Oberauer, L. Intense vacuum ultraviolet and infrared scintillation of liquid Ar-Xe mixtures. Europhysics Letters 109, (2015).

g] Piatek, S. What is an SiPM and how does it work?, https://hub.hamamatsu.com/jp/en/technical-note/how-sipm-works/index.htm.

10] Wahl, C. G. et al. Pulse-shape discrimination and energy resolution of a liquid ar

\section{Preliminary Work}

Fondazione Bruno Kessler Silicon Photomultipliers were tested using a SiPM Signal Processor to find their characteristics at room temperature and LAr temperatures using an $\mathrm{Am}^{241}$ source.
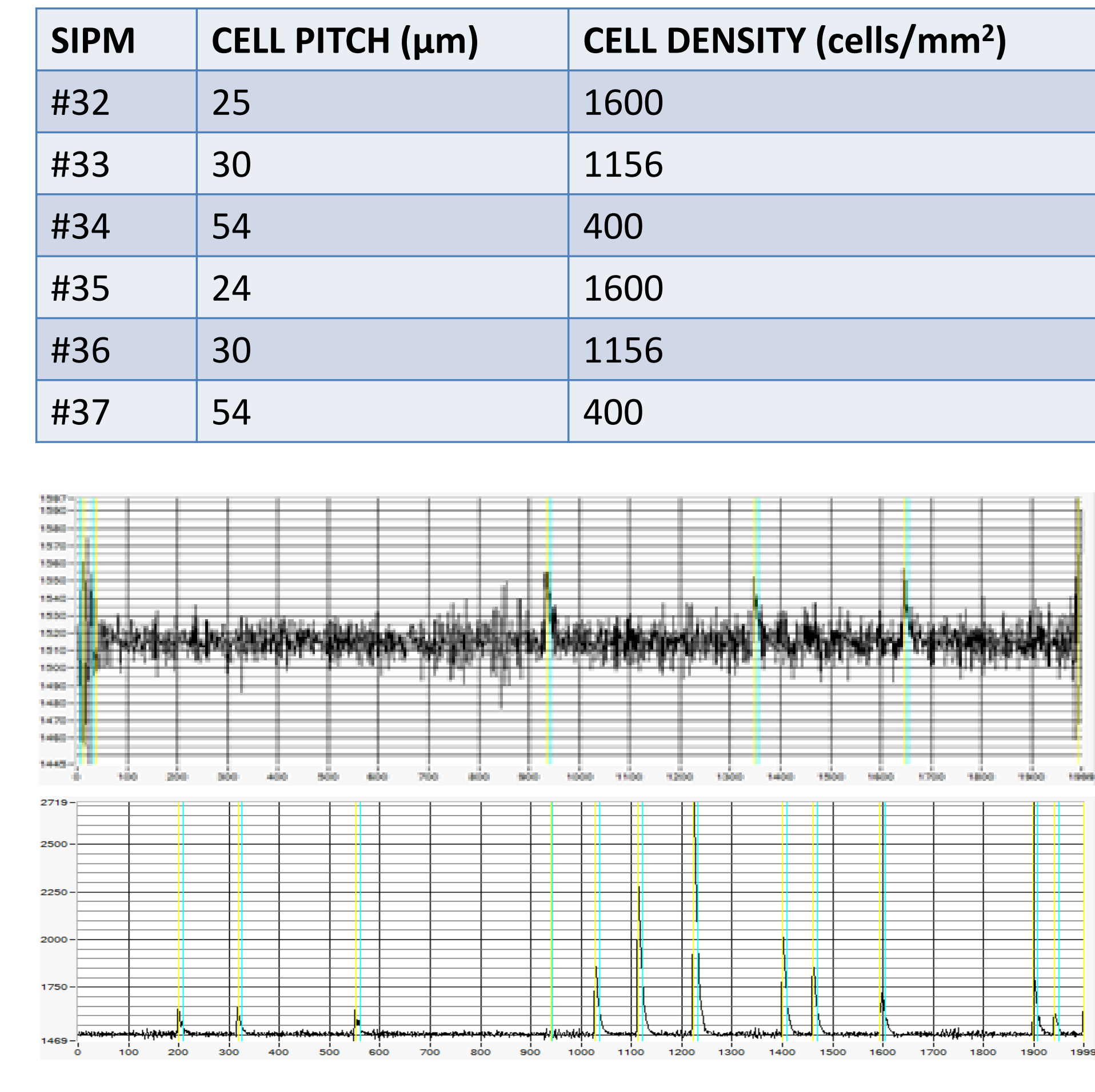

SiPM \#33

Liquid Argon Temperature: Only SiPM \#37 was tested at $87 \mathrm{~K}$. The following plots are the typical waveform in gaseous argon (top) and the typical waveform in liquid argon (bottom). A significant amount of light is present in liquid argon.

\section{Purity Test}

A light absorbing material named "MetalVelvet" was proposed to be utilized to absorb scattered light rays inside of the test chamber. MetalVelvet absorbs $99 \%$ of incident light over a wide range of wavelengths (from vacuum ultraviolet to infrared). Using this material along the inside of the test chamber would prevent reflected light from interacting with the detector and introducing systematic uncertainties in the data. MetalVelvet is not guaranteed to work at $84 \mathrm{~K}$, so it needed to be tested inside the cryostat LUKE.
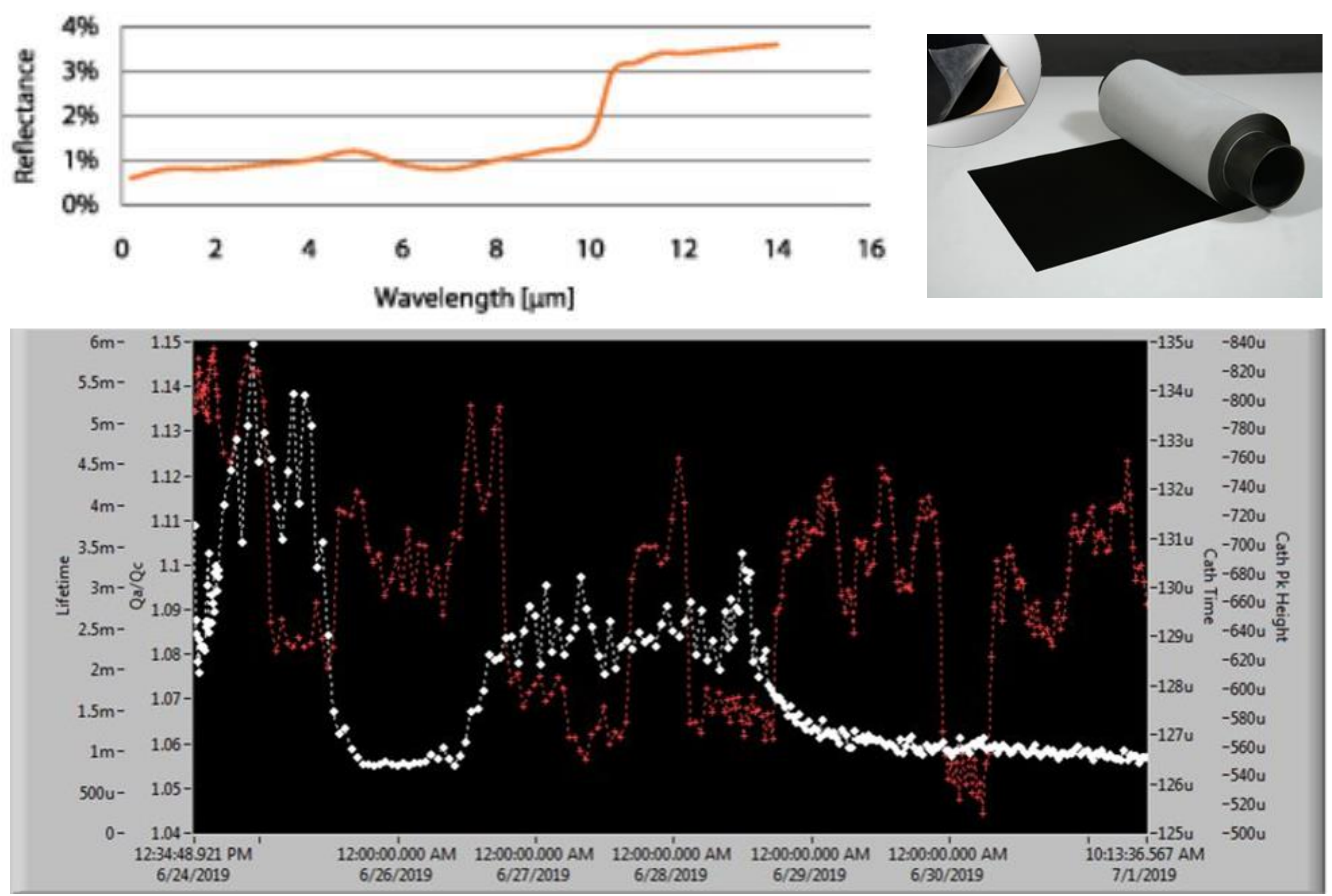

\section{Acknowledgements}

I would like to thank Alan Hahn for his guidance in executing the purity test; Paul Rubinov and Adam Para for their help with the SiPMs and electronics; William Miner and Ronald Davis for their assistance; and Carlos Escobar for allowing me to be a part of his research and supporting me along the way. 